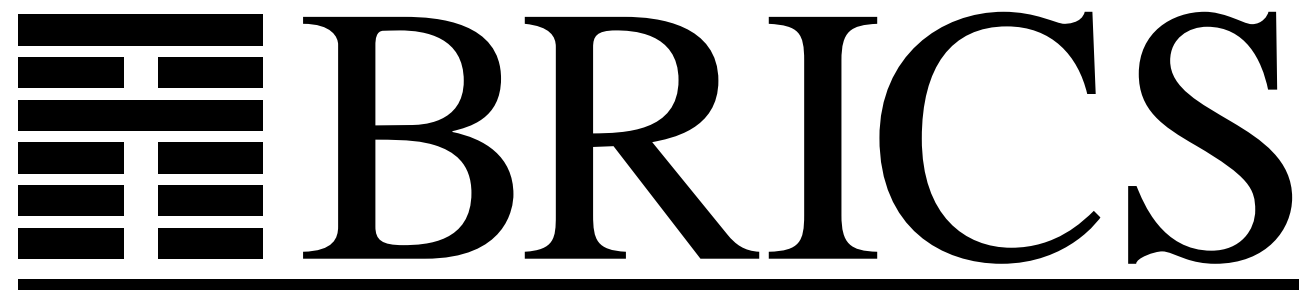

Basic Research in Computer Science

\title{
The Computational Strength of
} Extensions of Weak König's Lemma

Ulrich Kohlenbach

BRICS Report Series

RS-98-41 
Copyright (c) 1998, $\quad$ BRICS, Department of Computer Science University of Aarhus. All rights reserved.

Reproduction of all or part of this work is permitted for educational or research use on condition that this copyright notice is included in any copy.

See back inner page for a list of recent BRICS Report Series publications. Copies may be obtained by contacting:

\author{
BRICS \\ Department of Computer Science \\ University of Aarhus \\ Ny Munkegade, building 540 \\ DK-8000 Aarhus C \\ Denmark \\ Telephone: +4589423360 \\ Telefax: $\quad$ +45 89423255 \\ Internet: BRICS@brics.dk
}

BRICS publications are in general accessible through the World Wide Web and anonymous FTP through these URLs:

http://www.brics.dk

ftp://ftp.brics.dk

This document in subdirectory RS / $98 / 41 /$ 


\title{
The computational strength of extensions of weak König's lemma
}

\author{
Ulrich Kohlenbach \\ BRICS* \\ Department of Computer Science \\ University of Aarhus \\ Ny Munkegade \\ DK-8000 Aarhus C, Denmark \\ kohlenb@brics.dk
}

\begin{abstract}
The weak König's lemma WKL is of crucial significance in the study of fragments of mathematics which on the one hand are mathematically strong but on the other hand have a low proof-theoretic and computational strength. In addition to the restriction to binary trees (or equivalently bounded trees), WKL is also 'weak' in that the tree predicate is quantifier-free. Whereas in general the computational and proof-theoretic strength increases when logically more complex trees are allowed, we show that this is not the case for trees which are given by formulas in a class $\Phi_{\infty}$ where we allow an arbitrary function quantifier prefix over bounded functions in front of a $\Pi_{1}^{0}$-formula. This results in a schema $\Phi_{\infty}$-WKL.

Another way of looking at WKL is via its equivalence to the principle

$$
\forall x \exists y \leq 1 \forall z A_{0}(x, y, z) \rightarrow \exists f \leq \lambda x .1 \forall x, z A_{0}(x, f x, z),
$$

where $A_{0}$ is a quantifier-free formula ( $x, y, z$ are natural number variables). We generalize this to $\Phi_{\infty}$-formulas as well and allow function quantifiers ' $\exists g \leq s$ ' instead of ' $\exists y \leq 1$ ', where $g \leq s$ is defined pointwise. The resulting schema is
\end{abstract}

*Basic Research in Computer Science, Centre of the Danish National Research Foundation. 
called $\Phi_{\infty}$-b-AC $\mathrm{AC}^{0,1}$

In the absence of functional parameters (so in particular in a second order context), the corresponding versions of $\Phi_{\infty}$-WKL and $\Phi_{\infty}$-b-AC ${ }^{0,1}$ turn out to be equivalent to WKL. This changes completely in the presence of functional variables of type 2 where we get proper hierarchies of principles $\Phi_{n}$-WKL and $\Phi_{n}$-b-AC ${ }^{0,1}$. Variables of type 2 however are necessary for a direct representation of analytical objects and - sometimes - for a faithful representation of such objects at all as we will show in a subsequent paper. By a reduction of $\Phi_{\infty}$-WKL and $\Phi_{\infty}$-b-AC ${ }^{0,1}$ to a non-standard axiom $F$ (introduced in a previous paper) and a new elimination result for $F$ relative to various fragment of arithmetic in all finite types, we prove that $\Phi_{\infty}$-WKL and $\Phi_{\infty}$-b-AC ${ }^{0,1}$ do neither contribute to the provably recursive functionals of these fragments nor to their proof-theoretic strength. In a subsequent paper we will illustrate the greater mathematical strength of these principles (compared to WKL).

\section{Introduction}

The so-called weak König's lemma WKL is of crucial significance in the study of fragments of mathematics which on the one hand are mathematically strong but on the other hand have a low proof-theoretic and computational strength (see e.g. $[15],[10])$.

The prefix 'weak' has a twofold meaning: the full statement of König's lemma is restricted in the formulation of WKL in two ways

1) instead of allowing arbitrary finitely branching trees we only have binary trees in WKL (note however that it wouldn't make a difference if we would allow bounded trees in the sense of [15]);

2) the tree is represented by a function $f$ and consequently the tree predicate $f(n)=0$ expressing that $n$ is the code of a finite branch in the tree represented by $f$ is quantifier-free.

In view of 2), WKL could be denoted by QF-WKL, where 'QF' refers to 'quantifierfree'.

It is known that the removal of either of the restrictions above in general results in principles which, relative to certain weak subsystems of second order arithmetic or arithmetic in all finite types, are much stronger than WKL. E.g. the removal of 1) while keeping 2) would result in a principle called KL in the context of reverse mathematics which relative to the well-known system $\mathrm{RCA}_{0}$ is equivalent to arithmetical 
comprehension, whereas $\mathrm{RCA}_{0}+\mathrm{WKL}$ is conservative over $\mathrm{RCA}_{0}$ (see [15]).

The significance of the restriction 2) was pointed out first in [17], where it is shown that the binary König's lemma applied to trees of arbitrary logical complexity implies comprehension of numbers for arbitrary complex predicates.

In this paper we study an extension $\Phi_{\infty}$-WKL of (QF-)WKL to a certain class of formulas $\Phi_{\infty}$ which are built up as follows: in front of a $\Pi_{1}^{0}$-formula (with arbitrary parameters) we have an arbitrary prefix of bounded function quantifiers $' \forall(\exists) f \leq s$ ' (where $f \leq g: \equiv \forall x(f x \leq g x)){ }^{1}$

Another way of looking at WKL is to view it as a weak bounded choice principle. Indeed, already relative to very weak base systems, WKL is equivalent to

$$
\Pi_{1}^{0} \text {-b-AC }{ }^{0,0}: \forall x \exists y \leq 1 \forall z A_{0}(x, y, z) \rightarrow \exists f \leq \lambda x .1 \forall x, z A_{0}(x, f x, z),
$$

where $A_{0}$ is quantifier-free and $x, y, z$ are number variables.

We generalize this principle in two ways: we allow $\Phi_{\infty}$-formulas instead of the $\Pi_{1}^{0}$ formula ' $\forall z A_{0}(x, y, z)$ ' and generalize the existential number quantifier ' $\exists y \leq 1$ ' to a function quantifier ' $\exists g \leq \lambda x .1$ '. The resulting schema is called $\Phi_{\infty}$-b-AC ${ }^{0,1}$.

If no parameters of types $>1$ are present (so in particular in a second order context), the corresponding versions of $\Phi_{\infty}$-WKL and $\Phi_{\infty}$-b-AC ${ }^{0,1}$ turn out to be equivalent to WKL. This picture, however, changes drastically in the presence of higher type variables (actually the presence of parameters of type 2 suffices) where we get proper hierarchies of principles $\Phi_{n}$-WKL and $\Phi_{n}$-b-AC ${ }^{0,1}$. Variables of type 2 however are necessary for a direct representation of analytical objects and - sometimes - for a representation of such objects which is faithful at all. That is why we couldn't use WKL in our development of analysis in weak fragments of arithmetic in all finite types in [12],[13],[14], where e.g. continuous functions $\Phi: \mathbb{R} \rightarrow \mathbb{R}$ are represented directly as type-2-functionals and not as in the second order context of reverse mathematics ${ }^{2}$, but relied on certain non-standard principles $F$ and $F^{-}$instead which are not true in the full set-theoretic type structure but can be eliminated from proofs of sentences having a certain restricted logical form. In this paper we show that $\Phi_{\infty}$-WKL and $\Phi_{\infty}$-b-AC ${ }^{0,1}$ can be derived from $F$ and a schema of quantifier-free choice in higher types. Together with a new elimination result for $F$ this is used to calibrate the proof-theoretic and computational strength of $\Phi_{\infty}$-WKL and $\Phi_{\infty}$-b-AC ${ }^{0,1}$. It turns

\footnotetext{
${ }^{1}$ See definition 2.1 for a precise definition and also remark 2.2 .

${ }^{2}$ The need for a more flexible language than that of second-order arithmetic which includes variables of higher type for a direct formalization of analysis has been emphasized by S. Feferman in a number of writings, see e.g. [4].
} 
out that these principles do not contribute to the provably recursive function(al)s (up to type 2) of the underlying finite type systems we consider. Furthermore we obtain conservation results over primitive recursive arithmetic PRA resp. Peano arithmetic PA when these principles are added to the finite type versions of PRA and PA. The results of this paper can be understood also as an analysis of the greater mathematical strength of the non-standard principle $F$ (compared to WKL) in terms of standard extensions of WKL. That this greater strength is actually needed already for the treatment of continuous functions when the latter are represented as mentioned above, will be discussed in a subsequent paper.

\section{Description of the theories E-G $\mathbf{A}^{\omega},{\mathrm{E}-P R A^{\omega}}$ and E-PA ${ }^{\omega}$}

The set $\mathbf{T}$ of all finite types is defined inductively by

$$
\text { (i) } 0 \in \mathbf{T} \text { and }(i i) \rho, \tau \in \mathbf{T} \Rightarrow \tau(\rho) \in \mathbf{T} \text {. }
$$

Terms which denote a natural number have type 0 . Elements of type $\tau(\rho)$ are functions which map objects of type $\rho$ to objects of type $\tau$.

The set $\mathbf{P} \subset \mathbf{T}$ of pure types is defined by

$$
\text { (i) } 0 \in \mathbf{P} \text { and }(i i) \rho \in \mathbf{P} \Rightarrow 0(\rho) \in \mathbf{P} \text {. }
$$

Brackets whose occurrences are uniquely determined are often omitted, e.g. we write $0(00)$ instead of $0(0(0))$. Furthermore we write for short $\tau \rho_{k} \ldots \rho_{1}$ instead of $\tau\left(\rho_{k}\right) \ldots\left(\rho_{1}\right)$. Pure types can be represented by natural numbers: $0(n):=n+1$. The types $0,00,0(00), 0(0(00)) \ldots$ are so represented by $0,1,2,3 \ldots$ For arbitrary types $\rho \in \mathbf{T}$ the degree of $\rho$ (for $\operatorname{short} \operatorname{deg}(\rho)$ ) is defined by $\operatorname{deg}(0):=0$ and $\operatorname{deg}(\tau(\rho)):=\max (\operatorname{deg}(\tau), \operatorname{deg}(\rho)+1)$. For pure types the degree is just the number which represents this type.

Our theories $\mathcal{T}$ used in this paper are based on many-sorted classical logic formulated in the language of all finite types plus the combinators $\Pi_{\rho, \tau}, \Sigma_{\delta, \rho, \tau}$ which allow the definition of $\lambda$-abstraction.

The systems $\mathrm{E}_{-} \mathrm{G}_{n} \mathrm{~A}^{\omega}$ (for all $n \geq 1$ ) are introduced in [12] to which we refer for details. E- $\mathrm{G}_{n} \mathrm{~A}^{\omega}$ has as primitive relations $={ }_{0}, \leq_{0}$ for objects of type 0 , the constant $0^{0}$, functions $\min _{0}, \max _{0}, S^{00}$ (successor), $A_{0}, \ldots, A_{n}$, where $A_{i}$ is the $i$-th branch of the Ackermann function (i.e. $A_{0}(x, y)=y^{\prime}, A_{1}(x, y)=x+y, A_{2}(x, y)=x \cdot y, A_{3}(x, y)=$ $\left.x^{y}, \ldots\right)$, functionals of degree $2: \Phi_{1}, \ldots, \Phi_{n}$, where $\Phi_{1} f x=\max _{0}(f 0, \ldots, f x)$ and $\Phi_{i}$ is the iteration of $A_{i-1}$ on the $f$-values for $i \geq 2$, i.e. $\Phi_{2} f x=\sum_{i=0}^{x} f i, \Phi_{3} f x=$ 
$\prod_{i=0}^{x} f i, \ldots$ We also have a bounded search functional $\mu_{b}$ and bounded predicative recursion provided by recursor constants $\tilde{R}_{\rho}$ (where 'predicative' means that recursion is possible only at the type 0 as in the case of the (unbounded) Kleene-Feferman recursors $\widehat{R}_{\rho}$ ). In this paper our systems always contain the axioms of extensionality

$$
(E): \forall x^{\rho}, y^{\rho}, z^{\tau \rho}\left(x={ }_{\rho} y \rightarrow z x={ }_{\tau} z y\right)
$$

for all finite types $\left(x={ }_{\rho} y\right.$ is defined as $\forall z_{1}^{\rho_{1}}, \ldots, z_{k}^{\rho_{k}}\left(x z_{1} \ldots z_{k}={ }_{0} y z_{1} \ldots z_{k}\right)$ where $\left.\rho=0 \rho_{k} \ldots \rho_{1}\right)$.

In [12] we had in addition to the defining axioms for the constants of our theories all true sentences having the form $\forall x^{\rho} A_{0}(x)$, where $A_{0}$ is quantifier-free and $\operatorname{deg}(\rho) \leq 2$, added as axioms. ${ }^{3}$ By 'true' we refer to the full set-theoretic model $\mathcal{S}^{\omega}$. In given proofs of course only very special universal axioms are used which can be proved in suitable extensions of our theories. Nevertheless one can include them all as axioms since they (more precisely their proofs) do not contribute to the provable recursive function(al)s of the system. In particular this covers all instances of the schema of quantifier-free induction. In this paper we prefer however to have only the schema of quantifier-free choice included to $\mathrm{E}-\mathrm{G}_{n} \mathrm{~A}^{\omega}$ instead of taking arbitrary universal axioms. We note nevertheless that all results of this paper (except for the conservation results over PA and PRA at the end of the paper) remain valid if we add an arbitrary set of universal axioms to our systems.

$\mathrm{E}_{-\mathrm{PRA}}{ }^{\omega}$ results if we add the functional

$$
\Phi_{i t} 0 y f={ }_{0} y, \Phi_{i t} x^{\prime} y f={ }_{0} f\left(x, \Phi_{i t} x y f\right)
$$

to $E-G_{\infty} A^{\omega}:=\bigcup_{n \in \omega}\left\{E-G_{n} A^{\omega}\right\}$. The system E-PRA ${ }^{\omega}$ is equivalent to Feferman's system E-PA $\widehat{P A}^{\omega} \wedge$ from [3] since $\Phi_{i t}$ allows (relative to $\mathrm{E}-\mathrm{G}_{\infty} \mathrm{A}^{\omega}$ ) to define the predicative recursor constants $\widehat{R}_{\rho}$ (see [12]).

$\mathrm{E}_{-\mathrm{PA}^{\omega}}$ is the extension of E-PRA ${ }^{\omega}$ obtained by the addition of the schema of full induction and all (impredicative) primitive recursive functionals in the sense of [6].

The schema of full choice is given by

$$
\mathrm{AC}^{\rho, \tau}: \forall x^{\rho} \exists y^{\tau} A(x, y) \rightarrow \exists Y^{\tau(\rho)} \forall x^{\rho} A(x, Y x), \quad \mathrm{AC}:=\bigcup_{\rho, \tau \in \mathbf{T}}\left\{\mathrm{AC}^{\rho, \tau}\right\} .
$$

\footnotetext{
${ }^{3}$ The restriction $\operatorname{deg}(\rho) \leq 2$ has a technical reason discussed in [12].
} 
The schema of quantifier-free choice $\mathrm{QF}-\mathrm{AC}^{\rho, \tau}$ is defined as the restriction of $\mathrm{AC}^{\rho, \tau}$ to quantifier-free formulas $A_{0}{ }^{4}$

The theory $\mathcal{T}+\mu$ results from $\mathcal{T}$ if we add the non-constructive $\mu$-operator $\mu^{2}$ to $\mathcal{T}$ together with the characterizing axiom

$$
\mu(f)=\left\{\begin{array}{l}
\text { the least } x \text { such that } f(x)={ }_{0} 0, \text { if } \exists x^{0}\left(f(x)={ }_{0} 0\right) \\
0, \text { otherwise. }
\end{array}\right.
$$

Notation: For $\rho=0 \rho_{k} \ldots \rho_{1}$, we define $1^{\rho}:=\lambda x_{1}^{\rho_{1}} \ldots x_{k}^{\rho_{k}} \cdot 1^{0}$, where $1^{0}:=S 0$.

Definition 1.1 1) Between functionals of type $\rho$ we define the relation $\leq_{\rho}$ ('less or equal') by induction on the type:

$$
\left\{\begin{array}{l}
x_{1} \leq_{0} x_{2}: \equiv x_{1} \leq x_{2} \\
x_{1} \leq_{\tau \rho} x_{2}: \equiv \forall y^{\rho}\left(x_{1} y \leq_{\tau} x_{2} y\right)
\end{array}\right.
$$

2)

$$
\left\{\begin{array}{l}
\min _{0}\left(x_{1}^{0}, x_{2}^{0}\right):=\min \left(x_{1}, x_{2}\right), \\
\min _{\rho \tau}\left(x_{1}^{\rho \tau}, x_{2}^{\rho \tau}\right):=\lambda y^{\tau} \cdot \min _{\rho}\left(x_{1} y, x_{2} y\right) .
\end{array}\right.
$$

In the following we will need the definition of the binary ('weak') König's lemma as given in [17]:

\section{Definition 1.2 (Troelstra(74))}

WKL: $\equiv \forall f^{1}\left(T(f) \wedge \forall x^{0} \exists n^{0}\left(\right.\right.$ lth $\left.\left.n={ }_{0} x \wedge f n==_{0} 0\right) \rightarrow \exists b \leq_{1} \lambda k \cdot 1 \forall x^{0}\left(f(\bar{b} x)==_{0} 0\right)\right)$, where

$T f: \equiv \forall n^{0}, m^{0}\left(f(n * m)=_{0} 0 \rightarrow f n==_{0} 0\right) \wedge \forall n^{0}, x^{0}\left(f(n *\langle x\rangle)=_{0} 0 \rightarrow x \leq_{0} 1\right)$ (i.e. $T(f)$ asserts that $f$ represents a 0,1-tree).

\footnotetext{
${ }^{4}$ Throughout this paper $A_{0}, B_{0}, C_{0}, \ldots$ denote quantifier-free formulas.
} 


\section{Principles of bounded choice in higher types: $\Phi_{\infty}$-b- $\mathbf{A} \mathbf{C}^{0,1}$}

Definition 2.1 1) $A \in \Phi_{n}$ if

$$
A \equiv \forall f_{1} \leq_{1} s_{1}[\underline{a}] \exists f_{2} \leq_{1} s_{2}[\underline{a}] \ldots \forall^{(d)} f_{n} \leq_{1} s_{n}[\underline{a}] \forall x^{0} A_{0}\left(\underline{a}, f_{1}, \ldots, f_{n}, x\right),
$$

where $A_{0}$ is quantifier-free and $\underline{a}$ contains all free variables of $A$ and $s_{i}$ (which may have arbitrary types). The $f_{i}$ must not occur in $\underline{a}$.

2) $A \in \Psi_{n}$ if

$$
A \equiv \exists f_{1} \leq_{1} s_{1}[\underline{a}] \forall f_{2} \leq_{1} s_{2}[\underline{a}] \ldots \exists^{(d)} f_{n} \leq_{1} s_{n}[\underline{a}] \forall x^{0} A_{0}\left(\underline{a}, f_{1}, \ldots, f_{n}, x\right),
$$

where $A_{0}$ and $s_{i}$ as above.

3) The classes $\Phi_{n}^{-}$and $\Psi_{n}^{-}$result if we restrict ourselves to parameters $\underline{a}$ of type degree $\leq 1$ in $A_{0}$ and $s_{i}$.

Remark 2.2 One could also allow further universal number quantifiers $\forall x^{0}$ (but no existential quantifiers) to occur in between the bounded function quantifiers in the definition of $\Phi_{n}$. The results of this paper easily extend to this slightly generalized case. However, since our applications on which we will report in a subsequent paper do not need this we restrict ourselves to the definition of $\Phi_{n}$ as stated above in order to improve the readability of the proofs.

Remark 2.3 In the extensional context of our theories $\mathcal{T}$ we can code pairs of bounded function quantifiers of the same sort together:

$$
\forall f_{1} \leq_{1} s_{1} \forall f_{2} \leq_{1} s_{2} A\left(f_{1}, f_{2}\right) \leftrightarrow \forall f \leq_{1} j\left(s_{1}, s_{2}\right) A\left(\min _{1}\left(j_{1} f, s_{1}\right), \min _{1}\left(j_{2} f, s_{2}\right)\right)
$$

for some monotone function pairing as used e.g. in [12]. Analogously for $\exists f \leq_{1} s$.

\section{Definition 2.4}

$$
\Phi_{n} \text {-b-AC }^{\rho, \tau}: \forall a^{\tau \rho}\left(\forall x^{\rho} \exists y \leq_{\tau} a x A(x, y, a) \rightarrow \exists Y \leq_{\tau \rho} a \forall x^{\rho} A(x, Y x, a)\right),
$$

where $A \in \Phi_{n}$ (with arbitrary further parameters).

$\Psi_{n}$-b-AC ${ }^{\rho, \tau}$ (resp. $\Phi_{n}^{-}$-b-AC ${ }^{\rho, \tau}, \Psi_{n}^{-}$-b-AC ${ }^{\rho, \tau}$ ) are defined as $\Psi_{n}$-b-AC ${ }^{\rho, \tau}$ but with $A \in$ $\Psi_{n}\left(\right.$ resp. $\left.A \in \Phi_{n}^{-}, \Psi_{n}^{-}\right)$.

$\Phi_{\infty}^{(-)}-\mathrm{b}-\mathrm{AC}^{\rho, \tau}:=\bigcup_{n \in \omega} \Phi_{n}^{(-)} \mathrm{b}-\mathrm{AC}^{\rho, \tau}$. 
Remark 2.5 In $\mathcal{T}=\mathrm{E}_{-} \mathrm{G}_{3} \mathrm{~A}^{\omega}, \mathrm{E}_{-} \mathrm{PRA}^{\omega}$ and ${\mathrm{E}-\mathrm{PA}^{\omega}}^{\omega}$ the schema $\Phi_{n}$-b- $\mathrm{AC}^{\rho, \tau}$ can be written as a single axiom (for each fixed $n \in \mathbb{N}, \rho, \tau$ ) by replacing the quantifierfree matrix $A_{0}(x, y, a, \underline{v})$ of $A$ by ' $\Phi x y a \underline{v}={ }_{0} 0$ ', where $\Phi$ is a functional variable of suitable type, and replacing ' $f_{i} \leq_{1} s_{i}[\underline{a}]$ ' by ' $f_{i} \leq_{1} b_{i}$ ' for a free function variable $b_{i}$. This, however, is not possible for the restricted version $\Phi_{n}^{-}$-b- $\mathrm{AC}^{\rho, \tau}$.

The next proposition shows that in the absence of parameters of types $\geq 2$ (and so in particular in a second-order context) there is no point in considering $\Phi_{n}$-b- $\mathrm{AC}^{0,1}$ instead of $\Pi_{1}^{0}$-b-AC ${ }^{0,0}\left(=\Phi_{0}\right.$-b-AC $\left.{ }^{0,0}\right) .{ }^{5}$ For its proof we need the following

Lemma 2.6 Let $A_{0}\left(\underline{a}, g^{1}, y^{0}\right)$ be a quantifier-free formula of $\mathcal{T}:=\mathrm{E}_{-} \mathrm{G}_{3} \mathrm{~A}^{\omega}, \mathrm{E}-\mathrm{PRA}^{\omega}$ or E-PA ${ }^{\omega}$ containing (in addition to $g, y$ ) only parameters $\underline{a}$ of type levels $\leq 1$ and let $s$ be a term of $\mathcal{T}$ containing at most a as free variables. Then one can construct a $\Pi_{1}^{0}$-formula $B(\underline{a})$ of $\mathcal{T}$ (containing only $\underline{a}$ free) such that

$$
\mathcal{T}+\mathrm{WKL} \vdash \forall \underline{a}\left(B(\underline{a}) \leftrightarrow \exists g \leq_{1} s[\underline{a}] \forall y^{0} A_{0}(\underline{a}, g, y)\right) .
$$

Proof: For $\mathcal{T}=\mathrm{E}_{-} \mathrm{PRA}^{\omega}$ and $\mathcal{T}=\mathrm{E}-\mathrm{PA}^{\omega}$ this follows from (the proofs of) proposition 4.14 and corollary 4.15 in [10]. The use of the modulus $\tilde{t} x y k$ of pointwise continuity in $y$ used in the proof of proposition 4.14 in [10] can easily be replaced by a modulus $\widehat{t} x k$ of uniform continuity on $\left\{y: y \leq_{1} s x\right\}$. For closed $t \in \mathrm{E}-\mathrm{G}_{3} \mathrm{~A}^{\omega}$ such a modulus $\hat{t}$ can be constructed in $\mathrm{E}_{-} \mathrm{G}_{3} \mathrm{~A}^{\omega}$ by the method of [9] since the majorization argument used there is available in $\mathrm{E}_{-} \mathrm{G}_{3} \mathrm{~A}^{\omega}$ as was shown in [12].

Proposition 2.7 Let $m, n \geq 0$. Over $\mathcal{T}:=\mathrm{E}-\mathrm{G}_{3} \mathrm{~A}^{\omega}, \mathrm{E}_{-} \mathrm{PRA}^{\omega}$ or $\mathrm{E}^{-\mathrm{PA}^{\omega}}$ the following principles are equivalent:

(i) WKL,

(ii) $\Phi_{0}-\mathrm{b}-\mathrm{AC}^{0,0}$,

(iii) $\Phi_{m}^{-}-\mathrm{b}-\mathrm{AC}^{0,1}$,

(iv) $\Psi_{n}^{-}-\mathrm{b}-\mathrm{AC}^{0,1}$.

Proof: We first show the following Claim: Let $A(\underline{a})$ be a $\Phi_{n}^{-}$(or $\Psi_{n}^{-}$) formula containing only parameters $\underline{a}$ of type degree $\leq 1$. Then one can construct a $\Pi_{1}^{0}$-formula $B(\underline{a})$ such that

$$
\mathcal{T}+\mathrm{WKL} \vdash A(\underline{a}) \leftrightarrow B(\underline{a}) .
$$

\footnotetext{
${ }^{5}$ This is in sharp contrast to the case where arbitrary parameters are allowed as we will see below.
} 
Proof of the claim: We proceed by meta-induction on $n$ :

$n=0:$ In this case $A \in \Pi_{1}^{0}$ and so $B:=A$ suffices.

$n \rightarrow n+1:$ Case 1: $A \in \Phi_{n+1}$. Then $A(\underline{a}) \equiv \forall f \leq_{1} s[\underline{a}] \tilde{A}(\underline{a}, f)$, where $\tilde{A} \in \Psi_{n}$. By the induction hypothesis there exists a formula $\tilde{B}(\underline{a}, f) \equiv \forall y^{0} \tilde{B}_{0}(\underline{a}, f, y) \in \Pi_{1}^{0}$ with

$$
\mathcal{T}+\mathrm{WKL} \vdash A(\underline{a}) \leftrightarrow \forall f \leq_{1} s[\underline{a}] \forall y^{0} \tilde{B}_{0}(\underline{a}, f, y) .
$$

Let $t_{\tilde{B}_{0}}$ be a closed term of $\mathcal{T}$ such that

$$
\mathcal{T} \vdash \forall \underline{a}, f, y\left(t_{\tilde{B}_{0}}(\underline{a}, f, y)=_{0} 0 \leftrightarrow \tilde{B}_{0}(\underline{a}, f, y) .\right.
$$

From results in [9] (using for the case of $\mathrm{E}_{-} \mathrm{G}_{3} \mathrm{~A}^{\omega}$ also [12]) it follows that one can construct a closed term $\widehat{t}_{\tilde{B}_{0}}$ of $\mathcal{T}$ such that $\widehat{t}_{\tilde{B}_{0}}(\underline{a}, y)$ is (provably in $\mathcal{T}$ a modulus of uniform continuity for $\lambda f . t_{\tilde{B}_{0}}(\underline{a}, f, y)$ on $\left\{f: f \leq_{1} s[\underline{a}]\right\}$. Using this modulus, $\forall f \leq_{1} s[\underline{a}] \tilde{B}_{0}(\underline{a}, f, y)$ can be written as a quantifier-free formula and hence $\forall f \leq_{1} s[\underline{a}] \forall y \tilde{B}_{0}(\underline{a}, f, y)$ as a $\Pi_{1}^{0}$-formula $\widehat{B}(\underline{a})$. So

$$
\mathcal{T}+\mathrm{WKL} \vdash A(\underline{a}) \leftrightarrow \widehat{B}(\underline{a}) .
$$

Case 2: $A(\underline{a}) \in \Psi_{n+1}$. Then $A(\underline{a}) \equiv \exists f \leq_{1} s[\underline{a}] \tilde{A}(\underline{a}, f)$ with $\tilde{A}(\underline{a}, f) \in \Phi_{n}$. By I.H. there exists a formula $\tilde{B}(\underline{a}, f) \equiv \forall y^{0} \tilde{B}_{0}(\underline{a}, f, y) \in \Pi_{1}^{0}$ with

$$
\mathcal{T}+\mathrm{WKL} \vdash A(\underline{a}) \leftrightarrow \exists f \leq_{1} s[\underline{a}] \forall y^{0} \tilde{B}_{0}(\underline{a}, f, y) .
$$

By the lemma, there exists a $\Pi_{1}^{0}$-formula $\widehat{B}(\underline{a})$ such that

$$
\mathcal{T}+\mathrm{WKL} \vdash \widehat{B}(\underline{a}) \leftrightarrow \exists f \leq_{1} s[\underline{a}] \forall y^{0} \tilde{B}_{0}(\underline{a}, f, y) .
$$

So again

$$
\mathcal{T}+\mathrm{WKL} \vdash A(\underline{a}) \leftrightarrow \widehat{B}(\underline{a})
$$

with $\widehat{B} \in \Pi_{1}^{0}$.

This finishes the proof of the claim.

The claim implies that

$$
\mathcal{T}+\mathrm{WKL} \vdash \Phi_{m}^{-}-\mathrm{b}-\mathrm{AC}^{0,1} \leftrightarrow \Psi_{n}^{-} \text {-b-AC } \mathrm{A}^{0,1} \leftrightarrow \Phi_{0}^{-} \text {-b-AC } C^{0,1}
$$

for all $m, n \geq 0$. Also note that

$$
\begin{gathered}
\mathcal{T} \vdash \Phi_{0}-\mathrm{b}-\mathrm{AC}^{0,0} \leftrightarrow \Phi_{0}^{-}-\mathrm{b}-\mathrm{AC}^{0,0} \text { and } \\
\mathcal{T} \vdash \Phi_{0}^{-}-\mathrm{b}-\mathrm{AC}^{0,1} \rightarrow \Phi_{0}^{-} \text {-b- } \mathrm{AC}^{0,0} .
\end{gathered}
$$

Therefore it remains to show that 
a) $\mathcal{T} \vdash \Phi_{0}-\mathrm{b}-\mathrm{AC}^{0,0} \rightarrow \mathrm{WKL}$,

в) $\mathcal{T} \vdash \mathrm{WKL} \rightarrow \Phi_{0}^{-}-\mathrm{b}-\mathrm{AC}^{0,1}$

Proof of $\alpha$ ): Consider the formula ${ }^{6}$

$$
(+)\left\{\begin{array}{l}
\forall x^{0} \exists n \leq_{0} 1 \forall k>0(\exists m \leq \overline{1} k(l t h(m)=k \wedge f(x * m)=0) \\
\rightarrow \exists m \leq \overline{1}(k-1)(l \operatorname{th}(m)=k \cdot 1 \wedge f(x *\langle n\rangle * m)=0)) .
\end{array}\right.
$$

We now assume that $T(f)$ and first show that $(+)$ holds: Let $x$ be arbitrary but fixed. Case 1: $\forall k>0 \exists m \leq \overline{1} k(l t h(m)=k \wedge f(x * m)=0)$.

Then (using classical logic)

$$
\begin{aligned}
& \forall k>0 \exists m \leq \overline{1} k(l t h(m)=k \wedge f(x *\langle 0\rangle * m)=0) \vee \\
& \forall k>0 \exists m \leq \overline{1} k(l t h(m)=k \wedge f(x *\langle 1\rangle * m)=0) .
\end{aligned}
$$

In the case the first disjunct is true, choose $n=0$ and $n=1$ otherwise.

Case 2: $\exists k>0 \neg \exists m \leq \overline{1} k(l t h(m)=k \wedge f(x * m)=0)$. By the quantifier-free leastnumber-principle (hence by the schema QF-IA of quantifier-free induction) we find the least such $k$. Call it $k_{0}$.

2.1: $k_{0}=1$ : Choose $n \leq 1$ arbitrarily.

2.2: $k_{0}>1:$ Then

$$
\exists m \leq \overline{1}\left(k_{0}-1\right)\left(\operatorname{lth}(m)=k_{0}-1 \wedge f(x * m)=0\right) .
$$

choose $n:=(m)_{0}$ for such an $m$. This finishes the proof of $(+)$.

By $\Pi_{1}^{0}$-b-AC ${ }^{0,0}$ applied to $(+)$ we get a function $g$ such that

$$
\left\{\begin{array}{l}
\forall x^{0}\left(g x \leq_{0} 1 \wedge \forall k>0(\exists m \leq \overline{1} k(l t h(m)=k \wedge f(x * m)=0)\right. \\
\rightarrow \exists m \leq \overline{1}(k-1)(l t h(m)=k-1 \wedge f(x *\langle g x\rangle * m)=0))) .
\end{array}\right.
$$

Define $\tilde{h}(0):=\langle\rangle, \tilde{h}(n+1):=\tilde{h}(n) *\langle g(\tilde{h}(n))\rangle$.

The definition of $\tilde{h}$ can be carried out in $\mathrm{E}_{-} \mathrm{G}_{3} \mathrm{~A}^{\omega}$ using bounded recursion, since $g \leq 1$ and therefore $\tilde{h}(n) \leq \overline{1} n$ (by [12], $\Phi_{\langle\rangle} f x=\bar{f} x$ is definable in $\mathrm{E}_{-} \mathrm{G}_{3} \mathrm{~A}^{\omega}$ ).

\footnotetext{
${ }^{6}$ Here we use that our coding of finite sequences has the property that $\forall n, m, f, g(n \geq m \wedge \forall x(f x \geq g x) \rightarrow \bar{f} n \geq \bar{g} m)$, which is the case for the coding from [12].
} 
Now take $h(n):=(\tilde{h}(n+1))_{n}$. By quantifier-free induction we show that

$(++) \forall n(\tilde{h}(n)=\bar{h}(n))$ :

$n=0: \quad \tilde{h}(0)=\langle\rangle=\bar{h}(0)$.

$n \rightarrow n+1: \tilde{h}(n+1)=\tilde{h}(n) *\langle g(\tilde{h} n)\rangle \stackrel{\text { I.H. }}{=} \bar{h} n *\langle g(\tilde{h} n)\rangle \stackrel{\operatorname{lth}(\tilde{h} n)=n}{=} \bar{h}(n) *\left\langle(\tilde{h}(n+1))_{n}\right\rangle=$ $\bar{h}(n) *\langle h n\rangle=\bar{h}(n+1)$.

Let $k$ be arbitrary but fixed. We now show - again by quantifier-free induction on $n$ - that

$$
\forall n<k \exists m \leq \overline{1}(k \cdot n)(l t h(m)=k \cdot n \wedge f(\bar{h}(n) * m)=0):
$$

$n=0: \bar{h}(0) * m=m$, hence the claim follows from $T(f)$.

$n \rightarrow n+1$ : We may assume that $n+1<k$ : By I.H.

$$
\exists \tilde{m} \leq \overline{1}(k-n)(l t h(\tilde{m})=k \cdot n \wedge f(\bar{h}(n) * \tilde{m})=0) .
$$

Hence by $g$-definition

$$
\exists m \leq \overline{1}(k-(n+1))(l \operatorname{th}(m)=k \bullet(n+1) \wedge f(\underbrace{\bar{h} n *\langle g(\bar{h} n)\rangle}_{=\bar{h}(n+1)(++)} * m)=0),
$$

which is the claim for $n+1$.

So in total we have shown that $T(f)$ implies

$$
\forall k \forall n<k \exists m \leq \overline{1}(k-n)(l t h(m)=k \cdot n \wedge f(\bar{h}(n) * m)=0)
$$

and hence

$$
\forall n(f(\bar{h} n)=0)
$$

i.e. $h$ satisfies WKL.

Proof of $\beta$ : Let

$$
\forall a^{1(0)}\left(\forall x^{0} \exists y \leq_{1} a x \forall z^{0} A_{0}\left(x, y, z, a, \underline{b} \rightarrow \exists Y \leq_{1(0)} a \forall x^{0}, z^{0} A_{0}(x, Y x, z, a, \underline{b})\right)\right.
$$

be an instance of $\Phi_{0}^{-}$-b-AC $C^{0,1}$, where all additional parameters $\underline{b}$ have types of degree $\leq 1$. From (the proof of) proposition 4.14 in $[10]^{7}$ it follows (using the fact that the type $1(0)$ can be encoded into the type 1 ) that

$$
\begin{aligned}
(*) \mathcal{T}+\mathrm{WKL} \vdash & \forall a, \underline{b}\left(\exists Y \leq_{1(0)} a \forall x^{0}, z^{0} A_{0}(x, Y x, z, a, \underline{b}) \leftrightarrow\right. \\
& \left.\forall z^{0} \exists Y \leq_{1(0)} a \forall x^{0} \forall \tilde{z} \leq z A_{0}(x, Y x, \tilde{z}, a, \underline{b})\right) .
\end{aligned}
$$

\footnotetext{
${ }^{7}$ In [10] only the systems $\mathrm{PA}^{\omega}$ and $\mathrm{PRA}^{\omega}$ are treated. However all ingredients used in the proof of proposition 4.14 are also available for $\mathrm{E}-\mathrm{G}_{3} \mathrm{~A}^{\omega}$ (see [12]).
} 
Relative to $\mathcal{T}, \forall x \exists y \leq_{1} a x \forall z^{0} A_{0}(x, y, z, a, \underline{b})$ implies $\forall z \forall x \exists y \leq_{1} \quad a x \forall \tilde{z} \leq_{0} z A_{0}(x, y, \tilde{z}, a, \underline{b}) . \forall \tilde{z} \leq_{0} z A_{0}(x, y, \tilde{z}, a, \underline{b})$ can be written as a quantifier-free formula $\tilde{A}_{0}(x, y, z, a, \underline{b})$. Let $t_{\tilde{A}_{0}}$ be a closed term such that

$$
\mathcal{T} \vdash \forall x, y, z, a, \underline{b}\left(t_{\tilde{A}_{0}} x y z a \underline{b}={ }_{0} 0 \leftrightarrow \tilde{A}_{0}(x, y, z, a, \underline{b})\right) .
$$

As in the proof of the claim above, we use the fact that $t_{\tilde{A}_{0}}$ has a modulus of uniform continuity functional for $\lambda y^{1} \cdot t_{\tilde{A}_{0}} x y z a \underline{b}$ on $\left\{y: y \leq_{1} a x\right\}$ to replace ' $\exists y \leq_{1} a x$ ' by a finite disjunction. Bounded search then yields (relative to $\mathcal{T}$ ) $\left.\exists Y \leq_{1(0)} a \forall x \forall \tilde{z} \leq z A_{0}(x, Y x, \tilde{z}, a, b)\right)$. Together with $(*)$ above, this concludes the proof of $\beta$.

Remark 2.8 The equivalence of WKL and $\Phi_{0}-b-A C^{0,0}$ is closely related to the equivalence of WKL with the so-called $\Sigma_{1}^{0}$-separation principle which has been established relative to $R C A_{0}$ in the context of reverse mathematics (see [15]). The latter result in turn is closely related to theorem 6.1 of [7].

In the presence of higher type parameters the picture changes as we will show now.

Definition 2.9 We define the classes of formulas $\Pi_{n}^{1, b}$ and $\Psi_{n}^{1, b}$ simultaneously by induction on $n$ :

(i) $A \in \Pi_{0}^{1, b}=\Sigma_{0}^{1, b}$, if $A$ is quantifier-free;

(ii) if $A(f) \in \Pi_{n}^{1, b}$, then $\exists f \leq_{1} 1 A(f) \in \Sigma_{n+1}^{1, b}$;

(iii) if $A(f) \in \Sigma_{n}^{1, b}$, then $\forall f \leq_{1} 1 A(f) \in \Pi_{n+1}^{1, b}$.

A may contain arbitrary parameters (of arbitrary types).

Definition $2.10 \quad$ 1) The schema of $\Pi_{n}^{1, b}$-comprehension is given by

$$
\Pi_{n}^{1, b}-\mathrm{CA}: \exists g^{1} \forall x^{0}(g x=0 \leftrightarrow A(x)),
$$

where $A(x) \in \Pi_{n}^{1, b}$ and may contain arbitrary parameters (of arbitrary types) in addition to $x . \Sigma_{n}^{1, b}$-CA is defined analogously but with $\Sigma_{n}^{1, b}$ instead of $\Pi_{n}^{1, b}$.

2) The schema of $\Sigma_{n}^{1, b}$-choice is given by

$$
\Sigma_{n}^{1, b}-\mathrm{AC}: \forall x^{0} \exists f \leq_{1} 1 A(x, f) \rightarrow \exists g \leq_{1(0)} 1 A(x, g x),
$$

where $A(x, f) \in \Sigma_{n}^{1, b}$ and may contain arbitrary parameters. 
Proposition 2.11 Let $\mathcal{T}:=\mathrm{E}_{-} \mathrm{G}_{3} \mathrm{~A}^{\omega}$, E-PRA ${ }^{\omega}$ or E-PA ${ }^{\omega}$. Then

$$
\mathcal{T}+\Psi_{n+1}-\mathrm{b}-\mathrm{AC}^{0,0} \vdash \Pi_{n}^{1, b}-\mathrm{CA} .
$$

Proof: Apply $\Psi_{n+1}$-b-AC ${ }^{0,0}$ to $\forall x^{0} \exists y \leq_{0} 1(y=0 \leftrightarrow A(x))$, where $A(x) \in \Pi_{n}^{1, b}$ (note that $A(x)$ can be prenexed into formulas $\tilde{A}(x) \in \Phi_{n+1}$ and $\widehat{A} \in \Psi_{n+1}$ making use of remark 2.3).

Corollary to the proof: In proposition 2.11 actually the restriction of $\mathrm{b}-\mathrm{AC}^{0,0}$ to Boolean combinations of $\Phi_{n^{-}}$and $\Psi_{n}$-formulas instead of $\Psi_{n+1}$-formulas would have been sufficient.

Proposition 2.12 $\mathrm{E}-\mathrm{PA}^{\omega}+\Pi_{n}^{1, b}-\mathrm{CA}+\mu$ contains (modulo a canonical embedding which doesn't change the first order part) the second order system ( $\left.\Pi_{n}^{1}-\mathrm{CA}\right)$ known from reverse mathematics. ${ }^{8}$

Proof: Systems formulated in the language of second-order arithmetic with set variables like $\left(\Pi_{n}^{1}-\mathrm{CA}\right)$ can be embedded in (suitable) systems formulated in the language of functionals of all finite types by representing sets $X$ by their characteristic functions $\chi_{X}$ and replacing formulas ' $t \in X^{\prime}$ ' by ' $\chi_{X}(t)={ }_{0} 0$ '. In doing so and using the fact that the presence of $\mu$ allows to absorb an arbitrary arithmetical quantifier-prefix in front of a quantifier-free formula with arbitrary parameters uniformly in these parameters, the comprehension schema of $\left(\Pi_{n}^{1}\right.$-CA $)$ reduces to $\Pi_{n}^{1, b}-\mathrm{CA}$ above.

Together with the well-known fact that $\mathrm{E}-\mathrm{PA}^{\omega}+\mathrm{QF}-\mathrm{AC}^{1,0}+\mathrm{QF}-\mathrm{AC}^{0,1}+\mu$ can be reduced proof-theoretically to $\left(\Pi_{1}^{0}-\mathrm{CA}\right)_{<\varepsilon_{0}}{ }^{9}$ and hence is proof-theoretically much weaker than $\left(\Pi_{1}^{1}-\mathrm{CA}\right)$, it follows from propositions $2.11,2.12$ together with remark 2.5 that

Corollary 2.13 E-PA ${ }^{\omega}+\mathrm{QF}-\mathrm{AC}^{1,0}+\mathrm{QF}-\mathrm{AC}^{0,1}+\mu \nvdash \Psi_{2}-\mathrm{b}-\mathrm{AC}^{0,0}$.

Proposition 2.14 Let $\mathcal{T}:=\mathrm{E}_{-} \mathrm{G}_{3} \mathrm{~A}^{\omega}$, E-PRA ${ }^{\omega}$ or $\mathrm{E}-\mathrm{PA}^{\omega}$. Then

$$
\mathcal{T}+\Psi_{n}-\mathrm{b}-\mathrm{AC}^{0,1} \vdash \Sigma_{n}^{1, b}-\mathrm{AC} .
$$

Proof: Obvious.

Proposition 2.15 $\mathrm{E}-\mathrm{PA}^{\omega}+\Sigma_{n}^{1, b}-\mathrm{AC}+\mu$ contains (modulo the previously mentioned canonical embedding) the second order system $\left(\Sigma_{n}^{1}-\mathrm{AC}\right) .{ }^{10}$

Proof: Similar to the proof of proposition 2.12 .

\footnotetext{
${ }^{8}$ In the notation of [15], $\left(\Pi_{n}^{1}-\mathrm{CA}\right)$ is the system $\Pi_{n}^{1}-\mathrm{CA}_{0}+$ full induction.

${ }^{9}$ This follows from [3] together with elimination of extensionality (see also [1]).

${ }^{10}$ In the notation of [15] this system is $\Sigma_{n}^{1}-\mathrm{AC}_{0}+$ full induction.
} 


\section{Generalization of WKL to more complex trees: $\Phi_{\infty}$-WKL}

Definition 3.1 The generalization of WKL to $\Phi_{n}$-trees is given by

$$
\Phi_{n^{-}} \mathrm{WKL}: \forall n^{0} \exists f \leq{ }_{1} 1 \forall \tilde{n} \leq n A(\bar{f} \tilde{n}) \rightarrow \exists f \leq{ }_{1} 1 \forall n^{0} A(\bar{f} n),
$$

where $A\left(k^{0}\right) \in \Phi_{n}$ (with arbitrary further parameters of arbitrary types).

$\Psi_{n}$-WKL is defined analogously.

$\Phi_{\infty}-\mathrm{WKL}:=\bigcup_{n \in \omega}\left\{\Phi_{n}-\mathrm{WKL}\right\}$.

Remark 3.2 Like $\Phi_{n}$-b-AC ${ }^{\rho, \tau}$ (see remark 2.5) $\Phi_{n}$-WKL can be written as a single axiom for each fixed $n$.

Proposition 3.3 E- $\mathrm{G}_{3} \mathrm{~A}^{\omega} \vdash \mathrm{WKL} \leftrightarrow \Phi_{0^{-}} \mathrm{WKL} \leftrightarrow \Psi_{0^{-}}$WKL.

Proof: $\Phi_{0}$-WKL $\equiv \Psi_{0}$ WKL holds by definition. We have to show WKL $\leftrightarrow \Phi_{0^{-}}$WKL: The right-hand side obviously implies the left-hand side since $\Phi_{0}$-WKL allows the tree-predicate to be given even by a $\Pi_{1}^{0}$-formula whereas in WKL $T(f)$ is quantifierfree. So it remains to show that WKL $\rightarrow \Phi_{0}$-WKL: Assume

$$
(+) \forall n^{0} \exists g \leq_{1} 1 \forall \tilde{n} \leq n \forall z^{0} A_{0}(\bar{g} \tilde{n}, z) .
$$

Define $f$ such that

$$
(++) f(x)={ }_{0} 0 \leftrightarrow \forall i<\operatorname{lth}(x)\left((x)_{i} \leq 1\right) \wedge \forall \tilde{x} \sqsubseteq x \forall z \leq l \operatorname{th}(x) A_{0}(\tilde{x}, z),
$$

where ' $\tilde{x} \sqsubseteq x$ ' means that $\tilde{x}$ is the code of an initial segment of the sequence coded by $x$ (note that the right-hand side of $(++)$ can be written as a quantifier-free formula in $\left.\mathrm{E}-\mathrm{G}_{3} \mathrm{~A}^{\omega}\right)$.

$f$ satisfies $T(f)$ and - by $(+)$ - represents an infinite binary tree, i.e.

$$
\forall n \exists g \leq_{1} 1(f(\bar{g} n)=0) .
$$

Hence WKL yields

$$
\exists g \leq_{1} 1 \forall n(f(\bar{g} n)=0)
$$

which implies

$$
\exists g \leq_{1} 1 \forall n \forall m \leq n \forall z \leq n A_{0}(\bar{g} m, z),
$$

and therefore

$$
\exists g \leq_{1} 1 \forall n \forall z A_{0}(\bar{g} n, z) .
$$


Proposition 3.4 Let $\mathcal{T}:=\mathrm{E}-\mathrm{PA}^{\omega}$. Then

$$
\mathcal{T}+\Phi_{n+1}-\mathrm{WKL} \vdash \Pi_{n}^{1, b}-\mathrm{CA} .
$$

Proof: We use the following tree-predicate from [17]:

$$
\tilde{A}(k): \equiv\left\{\begin{array}{c}
(k)_{l t h(k) \dot{-1}} \leq 1 \wedge\left((k)_{l \operatorname{th}(k) \dot{-1}}=0 \rightarrow A(\operatorname{lth}(k) \dot{-1})\right) \wedge \\
\left.\left((k)_{\operatorname{lth}(k) \dot{-1}}=1 \rightarrow \neg A(\operatorname{lth}(k)-1)\right)\right), \text { if } l \operatorname{th}(k)>0 \\
\text { true, otherwise. }
\end{array}\right.
$$

For $A \in \Pi_{n}^{1, b}, \tilde{A}(k)$ can be written as a $\Phi_{n+1}$-formula (using remark 2.3). By induction on $n$ we can prove in $\mathrm{E}_{-} \mathrm{PA}^{\omega}$ that

$$
\forall n^{0} \exists f \leq_{1} 1 \forall \tilde{n} \leq n \tilde{A}(\bar{f} \tilde{n}) .
$$

$\Phi_{n+1}$-WKL therefore yields the characteristic function for $A(n)$.

Arguing as in the case of corollary 2.13 we obtain

Corollary 3.5 E-PA ${ }^{\omega}+\mathrm{QF}-\mathrm{AC}^{1,0}+\mathrm{QF}-\mathrm{AC}^{0,1}+\mu \not t \Phi_{2}-\mathrm{WKL}$.

\section{The computational strength of $\Phi_{\infty}$-b-AC $\mathbf{C}^{0,1}$ and $\Phi_{\infty}$-WKL}

In order to determine the effect (or rather non-effect as it will turn out) of $\Phi_{\infty}$-b-AC $\mathrm{C}^{0,1}$ and $\Phi_{\infty}$-WKL on the provably recursive functionals when added to $\mathcal{T}$, we make use of a certain non-standard axiom $F$ which was introduced first in $[12]^{11}$ (and has been applied e.g. in [14]):

$$
\boldsymbol{F}: \equiv \forall \Phi^{2(0)}, y^{1(0)} \exists y_{0} \leq_{1(0)} y \forall k^{0} \forall z \leq_{1} y k\left(\Phi k z \leq_{0} \Phi k\left(y_{0} k\right)\right) .
$$

We call this axiom 'non-standard' since it does not hold in the full set-theoretic type structure $\mathcal{S}^{\omega}$. Nevertheless its use can be eliminated from certain proofs thereby yielding classically true results. This has been discussed extensively in [12] to which we refer for further information. In that paper we mainly made use of a weaker version

\footnotetext{
${ }^{11} \mathrm{~A}$ special case of $F$ was studied already in [11] and called also $F$ in that paper but $F_{0}$ in [12].
} 
$F^{-}$of $F$ which allows a direct proof-theoretic elimination whereas our elimination of $F$ was based on a model-theoretic argument. In this paper however we need the full version $F$ and give a proof-theoretic reduction of the use of $F$ to a standard principle for certain formulas (see theorem 4.6 below). We apply $F$ via one of its consequences, the following principle of uniform $\Sigma_{1}^{0}$-boundedness:

Definition 4.1 ([12]) The schema $a^{12}$ of uniform $\Sigma_{1}^{0}$-boundedness is defined as

$$
\boldsymbol{\Sigma}_{1}^{0}-\mathbf{U B}:\left\{\begin{aligned}
\forall y^{1(0)}\left(\forall k^{0} \forall x \leq_{1} y k \exists z^{0} A(x, y, k, z)\right. \\
\left.\rightarrow \exists \chi^{1} \forall k^{0} \forall x \leq_{1} y k \exists z \leq_{0} \chi k A(x, y, k, z)\right)
\end{aligned}\right.
$$

where $A \equiv \exists \underline{l} A_{0}(\underline{l})$ and $\underline{l}$ is a tuple of variables of type 0 and $A_{0}$ is a quantifier-free formula (which may contain parameters of arbitrary types).

Proposition $4.2([12])$ Let $\mathcal{T}:=\mathrm{E}_{-} \mathrm{G}_{3} \mathrm{~A}^{\omega}, \mathrm{E}^{\mathrm{PRA}}{ }^{\omega}$ or $\mathrm{E}-\mathrm{PA}{ }^{\omega}$. Then $\mathcal{T}+\mathrm{QF}-\mathrm{AC}^{1,0}+F \vdash \Sigma_{1}^{0}-\mathrm{UB}$.

Proposition $4.3([12])$ E- $\mathrm{G}_{3} \mathrm{~A}^{\omega}+\Sigma_{1}^{0}-\mathrm{UB} \vdash \mathrm{WKL}$.

$\Sigma_{1}^{0}$-UB implies the existence of a modulus of uniform continuity for each extensional $\Phi^{1(1)}$ on $\left\{z^{1}: z \leq_{1} y\right\}$ (where 'continuity' refers to the usual metric on the Baire space $\left.\mathbb{N}^{\mathbb{N}}\right)$ :

Proposition $4.4([12])$

$$
\begin{aligned}
& \text { E-G } \mathrm{G}_{3} \mathrm{~A}^{\omega}+\Sigma_{1}^{0} \text {-UB } \vdash \\
& \forall \Phi^{1(1)} \forall y^{1} \exists \chi^{1} \forall k^{0} \forall z_{1}, z_{2} \leq_{1} y\left(\bigwedge_{i \leq_{0} \chi k}\left(z_{1} i={ }_{0} z_{2} i\right) \rightarrow \bigwedge_{j \leq 0}\left(\Phi z_{1} j={ }_{0} \Phi z_{2} j\right)\right) .
\end{aligned}
$$

Proposition 4.5 Let $\mathcal{T}:=\mathrm{E}-\mathrm{G}_{3} \mathrm{~A}^{\omega}, \mathrm{E}^{\mathrm{PRA}} \mathrm{A}^{\omega}$ or E-PA ${ }^{\omega}$. Then

$$
\mathcal{T}+\mathrm{QF}^{-\mathrm{AC}^{1,0}}+\mathrm{QF}^{-\mathrm{AC}^{0,1}}+F \vdash \Phi_{\infty} \text {-b-AC }{ }^{0,1}, \Phi_{\infty} \text {-WKL } .
$$

\footnotetext{
${ }^{12}$ Like $\Phi_{n}$-b-AC ${ }^{\rho, \tau}$ (see remark 2.5), $\Sigma_{1}^{0}$-UB can be written as a single axiom. However the schematic version is easier to apply.
} 
Proof: The idea of the proof is to use proposition 4.4 (together with propositions 4.2 and 4.3) to show similarly to the argument in the proof of proposition 2.7 that every $A \in \Phi_{n}$ (or $\in \Psi_{n}$ ) can be written as a $\Pi_{1}^{0}$-formula $B$. Whereas in the proof of proposition 2.7 we could use the fact that for every term $t^{2}[\underline{a}]$ of $\mathcal{T}$ containing only variables $\underline{a}$ of type $\leq 1$ one can construct a modulus of uniform continuity on $\left\{x: x \leq_{1} b\right\}$ (uniformly in $\underline{a}$ and $b$ ), we have to use proposition 4.4 in the presence of arbitrary parameters. The latter provides such a modulus of uniform continuity only uniformly in number parameters but not uniformly in function parameters $f$ unless the latter are themselves restricted to a compact set $\left\{f: f \leq_{1} b\right\}$ (in which case a modulus that is independent of $f$ does exist). However this is just the case in the situation at hand since all function variables $f_{1}, \ldots, f_{n}$ of $A \in \Phi_{n}$ which are not parameters are bounded and in the premise $\forall x^{0} \exists y \leq_{1}$ ax $A(x, y, a)$ of $\Phi_{n^{-}}$b-AC ${ }^{0,1}$ the function variable $y$ again is bounded. So all we need is

$$
(*)\left\{\begin{array}{l}
\forall \Phi, a^{1(0)}, \underline{a} \exists \alpha^{1} \forall x^{0}, z^{0}\left(\lambda y^{1}, \underline{f} .(\Phi x z y \underline{f} a \underline{a})^{0}\right. \text { is uniformly continuous for all } \\
\left.y \leq_{1} a x, f_{1} \leq_{1} s_{1}[x, y, a, \underline{a}], \ldots, f_{n} \leq_{1} s_{n}[x, y, a, \underline{a}] \text { with modulus } \alpha x z\right),
\end{array}\right.
$$

where $\underline{a}$ are all the remaining free variables of $s_{i}$ (which may have arbitrary types). ${ }^{13}$ Using $\Sigma_{1}^{0}$-UB one easily shows that

$$
\forall a^{1(0)}, \underline{a} \exists b^{1(0)} \forall x^{0} \forall y \leq_{1} a x\left(s_{i}[x, y, a, \underline{a}] \leq_{1} b x\right) .
$$

Hence $(*)$ is (in the presence of $\Sigma_{1}^{0}$-UB) implied by

$$
(* *)\left\{\begin{array}{l}
\forall \Phi, a^{1(0)}, \underline{b}^{1(0)} \exists \alpha^{1} \forall x^{0}, z^{0}\left(\lambda y^{1}, \underline{f} .(\Phi x z y \underline{f} a \underline{a})^{0}\right. \text { is uniformly continuous for all } \\
\left.y \leq_{1} a x, f_{1} \leq_{1} b_{1} x, \ldots, f_{n} \leq_{1} b_{n} x \text { with modulus } \alpha x z\right) .
\end{array}\right.
$$

But this follows in $\mathcal{T}+\Sigma_{1}^{0}$-UB (and therefore in $\mathcal{T}+\mathrm{QF}-\mathrm{AC}^{1,0}+F$ by proposition 4.2 ) similarly to the proof of proposition 4.4. Since by proposition 4.3 also WKL is available in this theory, we can argue as in the proof of the claim in the proof of proposition 2.7 and show that for $A(a, y, x) \in \Phi_{n}$ (with arbitrary additional parameters)

$$
\mathcal{T}+\Sigma_{1}^{0} \text {-UB } \vdash \forall a^{1(0)} \exists \Phi \forall x^{0} \forall y \leq_{1} a x\left(A(a, y, x) \leftrightarrow \forall z^{0}\left(\Phi a x z={ }_{0} 0\right)\right) .
$$

Hence

$$
(* * *) \mathcal{T}+\Sigma_{1}^{0} \text {-UB } \vdash \Phi_{0} \text {-b-AC }{ }^{0,1} \rightarrow \Phi_{n} \text {-b-AC }{ }^{0,1} .
$$

\footnotetext{
${ }^{13}$ Here ' $z$ ' is the variable from the $\Pi_{1}^{0}$-kernel of $A$ (which of course can be merged together with $x)$.
} 
We now show that $(* * * *) \mathcal{T}+\mathrm{QF}-\mathrm{AC}^{0,1}+\Sigma_{1}^{0}$-UB $\vdash \Phi_{0}$-b- $\mathrm{AC}^{0,1}$ :

Let

$$
\forall x^{0} \exists y \leq_{1} a x \forall z^{0} A_{0}(x, y, z) \rightarrow \exists Y \leq_{1(0)} a \forall x^{0}, z^{0} A_{0}(x, Y x, z)
$$

be an instance of $\Phi_{0}$-b-AC $\mathrm{C}^{0,1}$ and assume that

$$
\forall Y \leq_{1(0)} a \exists x^{0}, z^{0} \neg A_{0}(x, Y x, z) .
$$

By $\Sigma_{1}^{0}$-UB we obtain (using an encoding of 1(0) into type 1 )

$$
(+) \exists z^{0} \forall Y \leq_{1(0)} a \exists x \exists \tilde{z} \leq z \neg A_{0}(x, Y x, \tilde{z}) .
$$

However

$$
\forall x^{0} \exists y \leq_{1} a x \forall z^{0} A_{0}(x, y, z)
$$

yields

$$
\forall x^{0}, z^{0} \exists y \leq_{1} a x \forall \tilde{z} \leq z A_{0}(x, y, \tilde{z})
$$

and so - relative to $\mathcal{T}$ -

$$
\forall x^{0}, z^{0} \exists y^{1} \forall \tilde{z} \leq z A_{0}\left(x, \min _{1}(y, a x), \tilde{z}\right),
$$

which by $\mathrm{QF}-\mathrm{AC}^{0,1}$ yields a contradiction to $(+) .(* * *)$ and $(* * * *)$ together with proposition 4.2 imply

$$
\mathcal{T}+\mathrm{QF}-\mathrm{AC}^{1,0}+\mathrm{QF}-\mathrm{AC}^{0,1}+F \vdash \Phi_{n}-\mathrm{b}-\mathrm{AC}^{0,1}
$$

for every $n \in \mathbb{N}$.

Likewise as $(* * *)$, we obtain

$$
\mathcal{T}+\Sigma_{1}^{0}-\mathrm{UB} \vdash \Phi_{0}-\mathrm{WKL} \rightarrow \Phi_{n}-\mathrm{WKL}
$$

and hence (using propositions 4.3 and 3.3)

$$
\mathcal{T}+\Sigma_{1}^{0} \text {-UB } \vdash \Phi_{n^{-}} \text {WKL }
$$

and therefore by proposition 4.2

$$
\mathcal{T}+\mathrm{QF}-\mathrm{AC}^{1,0}+F \vdash \Phi_{n}-\mathrm{WKL},
$$

which concludes the proof.

Notation: For $f^{1}$ we define $f^{M}(x):=\max _{i \leq x} f(i)$. 
Theorem 4.6 Let $\forall f^{1}, x^{0} \exists y^{0} A_{0}(f, x, y)$ be a sentence of the language of $\mathcal{T}$ where $\mathcal{T}:=\mathrm{E}_{-} \mathrm{G}_{n} \mathrm{~A}^{\omega}(n \geq 2), \mathrm{E}^{-\mathrm{PRA}^{\omega}}$ or $\mathrm{E}-\mathrm{PA}^{\omega}$. Then the following rule holds

$$
\left\{\begin{array}{l}
\mathcal{T}+\text { QF-AC }{ }^{1,0}+\mathrm{QF}-\mathrm{AC}^{0,1}+F \vdash \forall f^{1}, x^{0} \exists y^{0} A_{0}(f, x, y) \\
\Rightarrow \text { one can extract a closed term } \Psi^{001} \text { of } \mathcal{T} \text { such that } \\
\tilde{\mathcal{T}} \vdash \forall f^{1}, x^{0} A_{0}(f, x, \Psi f x),
\end{array}\right.
$$

where

$$
\tilde{\mathcal{T}}:=\left\{\begin{array}{l}
\mathrm{E}^{-\mathrm{PA}^{\omega}}, \text { if } \mathcal{T}=\mathrm{E}^{-\mathrm{PA}}{ }^{\omega} \\
\mathrm{E}-\mathrm{PRA}^{\omega}, \text { if } \mathcal{T}=\mathrm{E}^{-\mathrm{G}_{n}} \mathrm{~A}^{\omega} \text { or E-PRA }{ }^{\omega} .
\end{array}\right.
$$

For $\mathcal{T}:=\mathrm{E}-\mathrm{G}_{2} \mathrm{~A}^{\omega}\left(\mathrm{E}-\mathrm{G}_{3} \mathrm{~A}^{\omega}, \mathrm{PRA}^{\omega}\right), \Psi$ is (provably in $\mathcal{T}$ ) bounded by a polynomial ${ }^{14}$ in $f^{M}, x$ ( $\Psi$ is an elementary recursive functional resp. a (Kleene-)primitive recursive functional).

\section{Proof:}

Extraction of $\Psi$ : The extractability of $\Psi$ from a proof of $\forall f, x \exists y A_{0}(f, x, y)$ in $\mathcal{T}+\mathrm{QF}_{-\mathrm{AC}}{ }^{1,0}+\mathrm{QF}-\mathrm{AC}{ }^{0,1}+F$ with a verification in $\mathcal{T}_{-E}^{i}+F^{D}$ by elimination of extensionality, negative translation and subsequent monotone functional interpretation follows from (the proof of) theorem 4.9 in [12]. Here

$$
F^{D}: \equiv \exists Y \leq_{\rho} \lambda \Phi, y \cdot y \forall \Phi^{2(0)}, y^{1(0)}, k^{0}, z^{1}\left(\Phi\left(k, \min _{1}(z, y k)\right) \leq_{0} \Phi(k, Y \Phi y k)\right) \text { and }
$$

$\mathcal{T}_{-E}^{i}$ results from $\mathcal{T}$ if we replace classical logic by intuitionistic logic and remove the extensionality axioms $(E)$ (except extensionality for numbers $x=_{0} y \rightarrow f x=_{0} f y$ which we keep). From [12] it follows that $\Psi$ is bounded by a polynomial in $f^{M}, x$ if $\mathcal{T}=\mathrm{E}-\mathrm{G}_{2} \mathrm{~A}^{\omega}$ and that $\Psi$ is elementary recursive (resp. (Kleene-)primitive recursive) if $\mathcal{T}=\mathrm{E}-\mathrm{G}_{3} \mathrm{~A}^{\omega}$ (resp. $\mathcal{T}=\mathrm{PRA}^{\omega}$ ). As already $F$ is not true in the full set-theoretic type structure $\mathcal{S}^{\omega}$ this holds a fortiori for $F^{D}$. However, we now give a verification by a principle $(*)$ that is classically true):

Classical verification of $\Psi$ : By the deduction theorem for $\mathcal{T}_{-E}^{i}$ and direct (i.e. without preceeding negative translation) monotone functional interpretation we can

\footnotetext{
${ }^{14}$ ' $\Psi f x$ is a polynomial in $f, x$ ' means that $\Psi f x$ can be written as a term $t[f, x]$ which is built up from $0, S,+, \cdot$ and $f, x$ only. See [12] for a discussion of this notion.
} 
extract a closed term $\chi$ of $\mathcal{T}$ such that

$$
\begin{aligned}
\mathcal{T}_{-E}^{i} & \vdash Y \leq_{\rho} \lambda \Phi, y . y, f^{1}, x^{0} \exists y^{1(0)}, k^{0}, z^{1} \\
& \left(\chi(Y, f, x)\left(k, \min _{1}(z, y k)\right) \leq_{0}(\chi(Y, f, x))(k, Y(\chi(Y, f, x)) y k) \rightarrow A_{0}(f, x, \Psi f x)\right) .
\end{aligned}
$$

Using Howard's majorizability as in [12] we can construct a closed term $\chi^{*}$ which majorizes $\chi$ (provably in $\mathcal{T}^{i}$ ) and consequently (using lemma 2.2.11 from [12])

$$
\mathcal{T}^{i} \vdash \forall Y \leq \lambda \Phi, y . y \forall f, x\left(\chi^{*}\left(\lambda \Phi, y . y, f^{M}, x\right) \operatorname{maj} \chi(Y, f, x)\right) .
$$

Hence

$$
\begin{aligned}
\mathcal{T}^{i} \vdash \exists Y \leq_{\rho} \lambda \Phi, y . y, f^{1}, x^{0} \forall \Phi\left(\Phi^{2(0)}\right. \text { majorizable } & \rightarrow \forall y^{1(0)}, k^{0}, z^{1} \\
\left.\left(\Phi\left(k, \min _{1}(z, y k)\right) \leq \Phi(k, Y \Phi y k)\right)\right) & \rightarrow \forall f, x A_{0}(f, x, \Psi f x) .
\end{aligned}
$$

Since ' $\Phi$ majorizable' implies that $\Phi k$ is bounded on $\left\{z: z \leq_{1} y k\right\}$ for all $k, y$ and using that $\min _{1}(z, y k) \leq_{1} y k$ we obtain

$$
\mathcal{T}^{i} \vdash(*) \rightarrow \forall f, x A_{0}(f, x, \Psi f x)
$$

where

$$
(*): \equiv\left\{\begin{aligned}
& \exists Y \leq_{\rho} \lambda \Phi, y \cdot y \forall \Phi^{2(0)}, y^{1(0)}, k^{0} \forall z \leq_{1} y k \\
&\left(\Phi k \text { bounded on }\left\{v: v \leq_{1} y k\right\} \rightarrow \Phi k z \leq_{0} \Phi(k, Y \Phi y k)\right)
\end{aligned}\right.
$$

with $\rho=1(0)(1(0))(2(0))$. (*) is classically true since $\mathrm{E}-\mathrm{PA}^{\omega}+\mathrm{AC} \vdash(*)$ :

By the least number principle we get

$$
\forall \Phi^{2(0)}, y^{1(0)}, k^{0}\left(\Phi k \text { bounded on }\left\{v: v \leq_{1} y k\right\} \rightarrow \exists v \leq_{1} y k \forall z \leq_{1} y k\left(\Phi k z \leq_{0} \Phi k v\right)\right)
$$

and hence by classical logic

$$
\forall \Phi^{2(0)}, y^{1(0)}, k^{0} \exists v \leq_{1} y k\left(\Phi k \text { bounded on }\left\{v: v \leq_{1} y k\right\} \rightarrow \forall z \leq_{1} y k\left(\Phi k z \leq_{0} \Phi k v\right)\right) .
$$

AC now yields $(*)$. A closer look at the proof of $(*)$ from $\mathrm{E}_{-\mathrm{PA}}{ }^{\omega}+\mathrm{AC}$ above shows that only an instance of bounded choice b-AC was used. Moreover this instance can be written using only bounded quantifiers (note that ' $\Phi k$ bounded on $\left\{v: v \leq_{1} y k\right\}$ ' is in fact equivalent (provably in E-PA ${ }^{\omega}$ ) to ' $\left.\exists v \leq_{1} y k \forall z \leq_{1} y k\left(\Phi k z \leq_{0} \Phi k v\right)^{\prime}\right)$ ). However 
we will not investigate this further but instead show that $(*)$ can be eliminated: Arithmetical verification of $\Psi$ : We show that E-PA ${ }^{\omega}+(*)\left(\right.$ resp. E-PRA $\left.{ }^{\omega}+(*)\right)$ is conservative over E-PA ${ }^{\omega}$ (resp. E-PRA ${ }^{\omega}$ ) w.r.t. sentences $\forall f^{1} A_{0}(f)$, where $A_{0}$ is quantifier-free (note that this concludes the proof of the theorem):

Similarly to the proof of $\widehat{F}$ from MUC in [11](p. 241) one shows that

$$
\mathrm{E}-\mathrm{PA}^{\omega}+\mathrm{MUC} \vdash(*),
$$

where

$$
\mathrm{MUC}: \equiv \exists \Omega^{3} \forall \Phi^{2} \forall y_{1}, y_{2} \leq_{1} 1\left(\bar{y}_{1}(\Omega \Phi)={ }_{0} \bar{y}_{2}(\Omega \Phi) \rightarrow \Phi y_{1}={ }_{0} \Phi y_{2}\right) .
$$

Hence E-PA ${ }^{\omega}+(*) \vdash \forall f^{1} A_{0}(f)$ implies that E-PA ${ }^{\omega}+\mathrm{MUC} \vdash \forall f^{1} A_{0}(f)$. By negative translation we obtain (using that the negative translation of MUC is intuitionistically implied by MUC)

$$
\text { E-HA }{ }^{\omega}+\mathrm{MUC} \vdash \forall f^{1} A_{0}(f),
$$

where E-HA ${ }^{\omega}$ is the intuitionistic version of E-PA ${ }^{\omega}$. Again as in [11](p. 241) we can conclude from there that

$$
\text { E-HA }{ }^{\omega} \vdash \forall f^{1} A_{0}(f) .
$$

It is not straightforward to see whether this last step which relies on non-trivial elimination arguments for choice sequences from [17] directly relativises to E-PRA ${ }^{\omega}$. That's why we give a different argument for the latter system (which can be adopted also for an alternative proof for the former):

In E-PRA ${ }^{\omega}+\Sigma_{1}^{0}$-IA one can show that the continuous functionals ECF form (pointwise) a model of E-PRA ${ }^{\omega}$. Moreover inspection of the proofs of 2.6.6,2.6.4 in [16] shows that E-PRA ${ }^{\omega}+\Sigma_{1}^{0}$-IA $+\Sigma_{1}^{0}$-UB $^{-} \vdash[\mathrm{MUC}]_{\mathrm{ECF}}$, where $\Sigma_{1}^{0}-\mathrm{UB}^{-}$is a restriction of $\Sigma_{1}^{0}$-UB (discussed in [12]) which allows a direct proof-theoretic elimination (see [12]). So we have

$$
\mathrm{E}_{-\mathrm{PRA}}{ }^{\omega}+\Sigma_{1}^{0}-\mathrm{IA}+\Sigma_{1}^{0} \mathrm{UB}^{-} \vdash\left[\forall f A_{0}(f)\right]_{\mathrm{ECF}}
$$

and therefore ${ }^{15}$

$$
\mathrm{E}_{-\mathrm{PRA}}{ }^{\omega}+\Sigma_{1}^{0} \mathrm{IA}+\Sigma_{1}^{0} \mathrm{UB}^{-} \vdash \forall f A_{0}(f) .
$$

By the elimination procedure for $\Sigma_{1}^{0} \mathrm{UB}^{-}\left([12]\left(\right.\right.$ thm.4.21)) and the fact that E-PRA ${ }^{\omega}+$ $\Sigma_{1}^{0}$-IA has (via elimination of extensionality and negative translation) a monotone functional interpretation in (E-)PRA ${ }^{\omega}$ we obtain that E-PRA ${ }^{\omega} \vdash \forall f A_{0}(f)$.

\footnotetext{
${ }^{15}$ Here we use that $\mathrm{E}_{-\mathrm{PRA}}{ }^{\omega} \vdash \Psi f^{1}{ }_{0} x \leftrightarrow[\Psi f]_{\mathrm{ECF}} \simeq x$ for closed terms $\Psi^{2}$ of E-PRA ${ }^{\omega}$. This however is easy since $\Psi f$ can be written as an ordinary primitive recursive functional in $f$ since no higher type recursion is present in E-PRA ${ }^{\omega}$.
} 
Corollary 4.7 1) $\mathrm{E}_{-\mathrm{PA}}{ }^{\omega}+\mathrm{QF}-\mathrm{AC}^{1,0}+\mathrm{QF}-\mathrm{AC}^{0,1}+\Phi_{\infty}-\mathrm{b}-\mathrm{AC}^{0,1}+\Phi_{\infty}-\mathrm{WKL}$ is conservative over $\mathrm{PA}$.

2) $\mathrm{E}_{-\mathrm{PRA}}{ }^{\omega}+\mathrm{QF}-\mathrm{AC}^{1,0}+\mathrm{QF}-\mathrm{AC}^{0,1}+\Phi_{\infty}$-b-AC $\mathrm{C}^{0,1}+\Phi_{\infty}$-WKL is $\Pi_{2}^{0}$-conservative over PRA.

Proof: 1) Let $A$ be a sentence of $\mathrm{PA}$ which is provable in $\mathrm{E}^{-\mathrm{PA}^{\omega}+\mathrm{QF}-\mathrm{AC}^{1,0}+\mathrm{QF}-}$

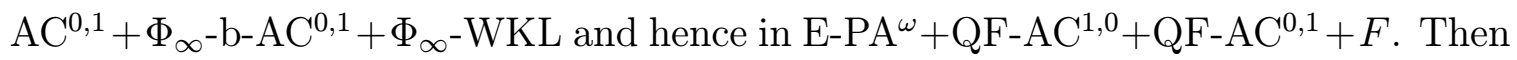
the Herbrand normal form $A^{H} \equiv \forall \underline{f} \exists \underline{y} A_{0}(\underline{f}, \underline{y})$ of $A$ is provable there a-fortiori. Hence by theorem 4.6

$$
\mathrm{E}-\mathrm{PA}^{\omega} \vdash \forall \underline{f} A_{0}(\underline{f}, \underline{\Psi}(\underline{f}))
$$

for suitable closed terms $\underline{\Psi}$ of E-PA ${ }^{\omega}$. Thus

$$
\mathrm{E}-\mathrm{PA}^{\omega} \vdash A^{H} \text {. }
$$

By $[8]\left(\right.$ thm.4.1) we can conclude that ${ }^{16}$

$$
\mathrm{PA} \vdash A \text {. }
$$

2) For $\Pi_{2}^{0}$-sentences $A$ the argument above relativises to E-PRA ${ }^{\omega}$ yielding E-PRA ${ }^{\omega} \vdash$ $A$. The conclusion now follows from the well-known fact that $\mathrm{E}_{-\mathrm{PRA}}{ }^{\omega}$ is $\Pi_{2}^{0}$-conservative over PRA.

\section{References}

[1] Avigad, J., Feferman, S., Gödel's functional ('Dialectica') interpretation. In: [2], pp. 337-405 (1998).

[2] Buss, S.R. (editor), Handbook of Proof Theory. Studies in Logic and the Foundations of Mathematics Vol 137, Elsevier, vii+811 pp. (1998).

[3] Feferman, S., Theories of finite type related to mathematical practice. In: Barwise, J. (ed.), Handbook of Mathematical Logic, pp. 913-972, North-Holland, Amsterdam (1977).

[4] Feferman, S., Infinity in mathematics: Is Cantor necessary?. In: G. Toraldo di Francia (ed.), L'infinito nella scienza, Istituto della Enciclopedia Italiana, Rome, pp. 151-209, 1987. Reprinted (with minor additions) in: [5].

\footnotetext{
${ }^{16}$ Warning: this argument does not apply to the subsystems E-PRA ${ }^{\omega}$, PRA; see [8] for a counterexample to this.
} 
[5] Feferman, S., In the Light of Logic. Oxford University Press, 340 pp. (1998).

[6] Gödel, K., Über eine bisher noch nicht benützte Erweiterung des finiten Standpunktes. Dialectica 12, pp. 280-287 (1958).

[7] Jockusch Jr., C.G., Soare, R.I., $\Pi_{1}^{0}$ classes and degrees of theories. Trans. AMS 173, pp. 35-56 (1972).

[8] Kohlenbach, U., Remarks on Herbrand normal forms and Herbrand realizations. Arch. Math. Logic 31, pp. 305-317 (1992).

[9] Kohlenbach, U., Pointwise hereditary majorization and some applications. Arch. Math. Logic 31, pp. 227-241 (1992).

[10] Kohlenbach, U., Effective bounds from ineffective proofs in analysis: an application of functional interpretation and majorization. J. Symbolic Logic 57, pp. 1239-1273 (1992).

[11] Kohlenbach, U., Analysing proofs in analysis. In: W. Hodges, M. Hyland, C. Steinhorn, J. Truss, editors, Logic: from Foundations to Applications. European Logic Colloquium (Keele, 1993), pp. 225-260, Oxford University Press (1996).

[12] Kohlenbach, U., Mathematically strong subsystems of analysis with low rate of growth of provably recursive functionals. Arch. Math. Logic 36, pp. 31-71 (1996).

[13] Kohlenbach, U., Proof theory and computational analysis. Electronic Notes in Theoretical Computer Science 13, Elsevier (http://www.elsevier.nl/locate/entcs/volume13.html), 1998.

[14] Kohlenbach, U., The use of a logical principle of uniform boundedness in analysis. To appear in: Proc. 'Logic in Florence 1995'.

[15] Simpson, S.G., Subsystems of Second Order Arithmetic. Perspectives in Mathematical Logic. Springer-Verlag. xiv+445 pp. 1999.

[16] Troelstra, A.S. (ed.) Metamathematical investigation of intuitionistic arithmetic and analysis. Springer Lecture Notes in Mathematics 344 (1973).

[17] Troelstra, A.S., Note on the fan theorem. J. Symbolic Logic 39, pp. 584-596 (1974). 


\section{Recent BRICS Report Series Publications}

RS-98-41 Ulrich Kohlenbach. The Computational Strength of Extensions of Weak König's Lemma. December 1998. 23 pp.

RS-98-40 Henrik Reif Andersen, Colin Stirling, and Glynn Winskel. A Compositional Proof System for the Modal $\mu$-Calculus. December 1998. 29 pp.

RS-98-39 Daniel Fridlender. An Interpretation of the Fan Theorem in Type Theory. December 1998. 15 pp. To appear in International Workshop on Types for Proofs and Programs 1998, TYPES 98 Selected Papers, LNCS, 1999.

RS-98-38 Daniel Fridlender and Mia Indrika. An n-ary zipwith in Haskell. December 1998. 12 pp.

RS-98-37 Ivan B. Damgård, Joe Kilian, and Louis Salvail. On the (Im)possibility of Basing Oblivious Transfer and Bit Commitment on Weakened Security Assumptions. December 1998. 22 pp. To appear in Advances in Cryptology: International Conference on the Theory and Application of Cryptographic Techniques, EUROCRYPT'99 Proceedings, LNCS, 1999.

RS-98-36 Ronald Cramer, Ivan B. Damgård, Stefan Dziembowski, Martin Hirt, and Tal Rabin. Efficient Multiparty Computations with Dishonest Minority. December 1998. 19 pp. To appear in Advances in Cryptology: International Conference on the Theory and Application of Cryptographic Techniques, EUROCRYPT'99 Proceedings, LNCS, 1999.

RS-98-35 Olivier Danvy and Zhe Yang. An Operational Investigation of the CPS Hierarchy. December 1998.

RS-98-34 Peter G. Binderup, Gudmund Skovbjerg Frandsen, Peter Bro Miltersen, and Sven Skyum. The Complexity of Identifying Large Equivalence Classes. December 1998. 15 pp.

RS-98-33 Hans Hüttel, Josva Kleist, Uwe Nestmann, and Massimo Merro. Migration $=$ Cloning $;$ Aliasing $($ Preliminary Version $)$. December 1998. 40 pp. To appear in 6th International Workshop on the Foundations of Object-Oriented, FOOL6 Informal Proceedings, 1998. 